\title{
Excursión diafragmática como predictor de éxito para el retiro de la ventilación mecánica en el paciente obeso
}

Diaphragmatic excursion as a predictor of success in the removal of mechanical ventilation in obese patient Mobilidade diafragmática como preditor de sucesso na remoção da ventilação mecânica no paciente obeso

\author{
Beatriz Valerio Munguía, ${ }^{*}$ Elizabeth Mendoza Portillo, ${ }^{\ddagger}$ Martín Mendoza Rodríguez ${ }^{*}$
}

\section{RESUMEN}

Introducción: El soporte respiratorio es una de las principales indicaciones para los pacientes que ingresan a la Unidad de Cuidados Intensivos (UCI). La liberación de la ventilación mecánica invasiva (VMI) representa hasta $40 \%$ del tiempo en la VMI; comorbilidades como obesidad son más frecuentes en la $\mathrm{UCl}$; existen recomendaciones en la liberación de la VMI en este grupo de pacientes, sin embargo, no existen predictores de éxito que esté ajustado para la población mexicana.

Objetivo: Evaluar la excursión diafragmática (ED) como predictor de éxito para el retiro de la VMI en pacientes obesos.

Material y métodos: Se realizó un estudio transversal, prospectivo, comparativo y analítico, en pacientes obesos ingresados a las $\mathrm{UCI}$ con VMI por más de 48 horas. Se analizaron variables demográficas, índice de ventilación de respiración superficial (IVRS) y ED. El punto de desenlace fue el éxito en la liberación de la VMI.

Resultados: La distribución por género fue $42 \%$ de mujeres y $58 \%$ de hombres; la edad fue de $41.3 \pm 12.8$ años; estancia hospitalaria de $9.6 \pm 4.6$ días; el tiempo de VMI: $8.8 \pm 4.8$ días, e índice de masa corporal (IMC): $33.3 \pm 2.7$ $\mathrm{kg} / \mathrm{cm}^{2}$; representados en grado I: $76 \%$, grado II: $2 \%$, grado III: $22 \%$, IVRS: $59 \pm 9.4$; ED: $1.55 \pm 0.11$. El área bajo la curva $(A B C)$ para IRS fue 0.60 y ED de 0.77 . Adicionalmente, el mejor punto de corte para éxito fue IVRS $<44$ con sensibilidad y especificidad de $100 \%$, y la ED $>1.9 \mathrm{~cm}$ con sensibilidad y especificidad de $100 \%$.

Conclusión: El mejor predictor de éxito fue la ED con respecto al IVRS. Se proponen nuevos puntos de corte para IVRS y ED.

Palabras clave: Índice de masa corporal, excursión diafragmática, destete.

\section{ABSTRACT}

Introduction: Respiratory support is an indication of admission to Intensive Care Units (ICU). The release of invasive mechanical ventilation (VMI), represents up to $40 \%$ of the time in VMI; comorbidities such as obesity are more frequent in the ICU; there are recommendations on the release of the $\mathrm{VMI}$ (LVMI) in this group of patients, however, there are no adjusted predictors of success for the Mexican population.

Objective: To evaluate the diaphragmatic excursion (ED) as a predictor of success in the removal of IMV in obese patients (PO).

Material and methods: A cross-sectional, prospective, comparative and analytical study was conducted in POs admitted to ICUs with IMV for more than 48 hours, demographic variables, surface respiration index (RSBI), ED were analyzed, the outcome was the success in LVMI.

Results: The distribution by gender was women $42 \%$ and men 58\%; age: 41.3 \pm 12.8 years; hospital stay: $9.6 \pm 4.6$ days; VMI time: $8.8 \pm 4.8$ days, I body mass index (BMI): $33.3 \pm 2.7$; represented in grade I: $76 \%$, grade $I: 2 \%$, grade III: $22 \%$, RSBI: $59 \pm 9.4$; ED: $1.55 \pm 0.11$. The area under the curve $(A B C)$ for $R S B I$ was 0.60 and ED 0.77. Additionally, the best cut-off point for success was: $R S B I<44$ with $100 \%$ sensitivity and specificity, and ED $>1.9 \mathrm{~cm}$ with $100 \%$ sensitivity and specificity.

Conclusion: The best predictor of success was the ED with respect to the $R S B I$, new cut-off points for RSBI and ED are proposed.

Keywords: Body mass index, diaphragmatic excursion, weaning.

\section{RESUMO}

Introdução: O suporte respiratório é uma indicação de admissão em Unidades de Terapia Intensiva (UTI), a liberação de ventilação mecânica invasiva (VMI) representa até $40 \%$ do tempo na VMI; comorbidades como obesidade são mais frequentes na UTI; existem recomendações sobre a liberação do VMI (LVMI)

\footnotetext{
* Hospital General La Villa, Secretaría de Salud, Ciudad de México, México.

‡ Hospital General «Dr. Rubén Leñero», Secretaría de Salud, Ciudad de México, México.
}

Recepción: 30/08/2019. Aceptación: 04/02/2020.

www.medigraphic.com/medicinacritica nesse grupo de pacientes, no entanto, não há preditores ajustados de sucesso para a população mexicana.

Objetivo: Avaliar a mobilidade diafragmática $(M D)$ como preditor de sucesso na remoção de VMI em pacientes obesos (PO).

Material e métodos: Foi realizado um estudo transversal, prospectivo, comparativo e analítico em PO admitidos em UTI com VMI por mais de 48 horas, foram analisadas variáveis demográficas, índice de ventilação da respiração superficial (IVRS), MD. O resultado foi bem-sucedido em LVMI.

Resultados: A distribuição por gênero foi de mulheres $42 \%$, homens $58 \%$; idade $41.3 \pm 12.8$ anos internação $9.6 \pm 4.6$ dias, tempo de VMI: $8.8 \pm 4.8$ dias, índice de massa corporal (IMC) 33.3 \pm 2.7 ; representado em Grau I 76\%, Grau II $2 \%$, Grau III 22\%, IRS $59 \pm 9.4 ;$ MD 1.55 \pm 0.11. A área sob a curva ( $A B C$ ) para IRS foi de 0.60 e MD 0.77. Além disso, o melhor ponto de corte para o sucesso foi: IVRS < $44 \mathrm{com} 100 \%$ de sensibilidade e especificidade e ED $>1.9 \mathrm{~cm} \mathrm{com}$ sensibilidade e especificidade de $100 \%$.

Conclusão: O melhor preditor de sucesso foi o MD em relação ao IVRS, novos pontos de corte para IVRS e MD são propostos.

Palavras-chave: Índice de massa corporal, mobilidade diafragmática, desmame.

\section{INTRODUCCIÓN}

El apoyo ventilatorio es una de las principales indicaciones para los pacientes que ingresan a la Unidad de Cuidados Intensivos (UCI). En la actualidad, no existen pautas específicas para liberar a un paciente en poblaciones especiales, como lo es el paciente obeso con ventilación mecánica invasiva (VMI). Frecuentemente, sólo se aplican de manera general, por lo que un protocolo de destete modificado a este tipo de pacientes puede ser útil, además de que tiene un impacto en el ahorro de recursos. ${ }^{1}$

La obesidad es una comorbilidad común en las UCI, presente en aproximadamente $20 \%$ de los pacientes, y el aumento de su incidencia se describe en todo el mundo. De acuerdo con la Organización Mundial de la Salud, ésta se define como un índice de masa corporal $(\mathrm{IMC}) \geq 30 \mathrm{~kg} / \mathrm{m}^{2}$. En las $\mathrm{UCl}$, se asocia con una mayor morbilidad, pero sigue habiendo incertidumbre con respecto al efecto sobre la mortalidad. En este sentido, distintos autores han descrito tasas de mortalidad similares al comparar pacientes con UCl obesos y no obesos. $^{2}$

El sistema respiratorio es uno de los objetivos capitales en el manejo de cuidados críticos en esta población, siendo el principal la prevención de complicaciones respiratorias. El manejo respiratorio de pacientes obesos en la $\mathrm{UCI}$ puede diferir entre pacientes con pulmones sanos y aquéllos con comorbilidades. ${ }^{3}$ En general, suele producir alteraciones anatómicas y fisiológicas que pueden afectar la función del sistema pulmonar en la cara, el cuello, la faringe, los pulmones y la pared to- 
rácica. ${ }^{4}$ El consumo de oxígeno, la producción de dióxido de carbono, el trabajo de respiración y la presión abdominal aumentan en pacientes obesos, mientras que el cumplimiento del sistema respiratorio y la capacidad residual funcional disminuyen. Es un factor de riesgo importante del síndrome de apnea obstructiva, el síndrome de insuficiencia respiratoria aguda (SIRA), complicación mortal cuyo pronóstico suele ser malo, además del consumo de más recursos para su tratamiento. ${ }^{3,5}$

La liberación de la ventilación mecánica es un proceso de dos pasos que implica, primero, una prueba de preparación para la extubación y, posteriormente, una segunda prueba de respiración espontánea. ${ }^{6}$ Los pacientes para considerarse listos para la extubación deben tener un adecuado nivel de consciencia, la mejoría o resolución de la enfermedad subyacente, la estabilidad hemodinámica frente a los vasopresores y una oxigenación adecuada con bajos requisitos de $\mathrm{FiO}_{2}(\leq 0.4)$ y PEEP $(\leq 5)$. El destete se realiza con mayor frecuencia mediante ensayos con tubo en $T$ o ventilación con soporte de presión, aunque es aceptable realizar una prueba de respiración espontánea por día de 30 a 120 minutos de duración. Los pacientes que toleran la prueba de respiración espontánea deben ser extubados, pero aquéllos que fallan en la prueba de respiración espontánea deben regresar a ventilación mecánica. ${ }^{3,7}$

La falla en la extubación se ha documentado que sucede entre 20 y $25 \%$, la cual genera mayores complicaciones como neumonía, traqueostomía, obstrucción de la vía aérea, broncoaspiración, complicaciones asociadas con una tasa de mortalidad significativamente más alta que aquéllos que se extuban con éxito en el primer intento. $^{8}$

En estos pacientes se ha registrado la disfunción diafragmática, la cual se define como la pérdida de la capacidad para generar una contracción adecuada, secundaria a debilidad y atrofia, que se asocia con la ventilación mecánica. ${ }^{8,9}$

El diafragma separa la cavidad torácica de la cavidad abdominal; es el principal músculo respiratorio y es inervado por los nervios frénicos que surgen del nervio de las raíces C3-C5. Sin embargo, la inervación, propiedades musculares contráctiles o mecánicas al acoplamiento de la pared torácica pueden causar disfunción diafragma, además de atrofia, la cual prolonga la duración de VMI. ${ }^{10,11}$

En este entorno, la ultrasonografía (US) es de gran importancia para valorar el momento ideal de VMI, la cual se realiza en la cabecera del paciente. Ésta resulta ser un método simple, portátil, rápido de realizarse, no invasivo y bien tolerado, con una relación entre el movimiento diafragmático y un volumen inspirado, que permite la evaluación del movimiento diafragmático y excursión diafragmática, además de que permite la visualización directa del espesor del diafragma en su zona de aposición. ${ }^{4,12}$ No hay exposición a radiación ionizante y la cooperación del paciente no es esencial; en manos expertas y siguiendo la metodología apropiada, es una técnica muy reproducible, con una buena confiabilidad y reproducibilidad, con alta sensibilidad (93\%) y especificidad (100\%) para la enfermedad neuromuscular diafragmática. ${ }^{10,13}$

El grosor del diafragma se puede determinar en más de $85 \%$ de las mediciones, con un bajo coeficiente de variación (0.09-0.14). La US se realiza con transductor de $3.5-5 \mathrm{MHz}$; ésta se posiciona inmediatamente de bajo del reborde costal derecho e izquierdo a la altura de la línea media clavicular o axilar anterior, dirigido en sentido craneal, medial y dorsal, por lo que el haz de la US alcanza perpendicularmente el tercio posterior del hemidiafragma. ${ }^{14}$ Se realiza un escaneo en eje largo de los espacios intercostales, con el lóbulo derecho del hígado como ventana acústica en el hemidiafragma derecho y el bazo al lado izquierdo. Se recomienda evaluar todas las vistas diafragmáticas que incluyen: vista intercostal y vista anterior subcostal, posterior subcostal y subxifoidea. El movimiento normal diafragmático durante la inspiración es caudal, ya que el diafragma se acerca a la sonda, y durante la espiración, el diafragma se aleja del transductor. ${ }^{15,16}$ Los pacientes se examinan en respiración espontánea; en pacientes que están bajo ventilación mecánica, se requiere desconectar brevemente del ventilador para visualizar mejor los esfuerzos respiratorios espontáneos. ${ }^{17}$

La excursión diafragmática (ED) puede evaluar en la vista anterior, posterior o subxifoidea, en modo movimiento (M) del US. En individuos sanos, se ha reportado un valor medio de $1.8 \pm 0.3 \mathrm{~cm}$ para hombres y para mujeres de $1.6 \pm 0.3 \mathrm{~cm}$, valores identificados por Kim y colaboradores $(>1.8 \mathrm{~cm}$ ) en pacientes ventilados en maniobras de retiro de ventilación con éxito. En varios estudios, no se ha identificado diferencias significativas en ambos hemidiafragmas. ${ }^{18}$

\section{MATERIAL Y MÉTODOS}

Se realizó un estudio descriptivo, transversal, prospectivo comparativo y analítico. En él, se calculó un número de muestra de 31 pacientes obesos mayores de 18 años, con una estancia mayor de 48 horas de apoyo ventilatorio y que hubieron ingresado a la $\mathrm{UCI}$ durante al menos cinco meses.

Los pacientes que no se incluyeron fueron aquellas obesas embarazadas, con neumopatía crónica previa, en los que se hubiera usado relajación muscular, aquéllos que ingresaron a la UCI con traqueostomía o que tuvieron enfermedades neuromusculares. Se eliminaron los que fueron trasladados a otra unidad médica, con 
alta voluntaria, con extubación accidental, o que fallecieran durante el estudio.

Los pacientes incluidos se agruparon de acuerdo con el índice de masa corporal (IMC), utilizando para ello la clasificación de la OMS; se registraron tanto los datos demográficos como los clínicos. A saber: género, edad, diagnósticos de ingreso a la $\mathrm{UCl}$, días de estancia en el servicio, días de apoyo ventilatorio y pruebas clásicas predictoras del retiro de la VMI. Asimismo, se midió la excursión diafragmática al completar el protocolo de destete y se evaluó como punto de desenlace el éxito o fracaso de la liberación de la VMI. Los pacientes se evaluaron durante su estancia en la $\mathrm{UCI}$ hasta completar el protocolo de destete para el retiro de la ventilación. Los datos se procesaron mediante el programa SPSS versión 24.

Finalmente, se evaluaron las características generales de los pacientes, destacando variables epidemiológicas mediante estadística descriptiva: proporciones, porcentajes y medidas de tendencia central. Se evaluaron las características de distribución de probabilidad, y para los puntos finales del estudio se determinaron, sensibilidad, especificada y curva ROC de los predictores del retiro de la ventilación mecánica.

\section{RESULTADOS}

En las mujeres el promedio de edad fue de $41.38 \pm$ 12.89 años. La distribución por género fue mujeres $(42 \%)$ y hombres $(58 \%)$ con base en el IMC, fue la siguiente: grado I de $30-34.9 \mathrm{~kg} / \mathrm{m}^{2} 26(76 \%)$, grado II de $35-39.9 \mathrm{~kg} / \mathrm{m}^{2}$ (2\%), grado III > $40 \mathrm{~kg} / \mathrm{m}^{2} 4(22 \%)$; con un promedio de $33.3 \pm 2.79 \mathrm{~kg} / \mathrm{m}^{2}$. En cuanto al tipo de paciente, en la mayoría de los grupos, éste fue de tipo quirúrgico, con 16 pacientes (51.61\%). La cantidad de días de estancia hospitalaria en la UCI fue en el $70.97 \%$ de 5 a 10 días, seguida de $12.9 \%$ de 11 a16 días, $12.9 \%$ de 17 a 22 días, y finalmente, en el 3\% de 29 a 34 días. La principal complicación que se presentó en la totalidad de la población y por estratificación por sexos fue la lesión renal aguda (52\%), seguida de la neumonía adquirida por ventilación (36.4\%); lesión renal aguda y neumonía adquirida por ventilación (7\%), y finalmente, traqueostomía (4.6\%). Al completar el protocolo de destete, el IVRS promedio fue de $59.00 \pm 9.4$, mientras que la excursión diafragmática fue de $1.55 \pm 0.11$.

La excursión diafragmática en la población total se encontró en $1.5 \mathrm{~cm}$ (0.3) (p50): en los hombres en 1.6 $\mathrm{cm}$, y en las mujeres en 1.4 centímetros.

Para la extubación exitosa, se reportaron 28 casos; de los cuales, $60 \%$ fue en hombres, con una edad promedio de 41.8 años (p50). El IMC fue muy similar entre ambos sexos, encontrándose un aumento en la categoría 1 en los que tuvieron extubación fallida (100\%). En la extubación exitosa, la causa principal fue la qui- rúrgica $(62.3 \%)$ en comparación con la fallida, que fue la médica (80\%). En la figura 1 se muestra el total de la población exitosa fue $90 \%$, mientras que $10 \%$ fue fallida, sin presentar complicaciones posterior a la extubación.

Al efectuar los análisis ROC con la excursión diafragmática y el índice IVRS para hacer el diagnóstico de extubación exitosa en pacientes obesos con VMI (Figura 2), pudimos observar que la medición de la excursión diafragmática es el mejor indicador para realizar dicho diagnóstico (AUC 0.77) en comparación con el índice de ventilación rápida y superficial (IVRS) (AUC 0.60); adicionalmente, se buscó en mejor punto de corte para éxito el mejor punto de corte para éxito: IVRS $<44$ con sensibilidad y especificidad $100 \%$, y la ED $>1.9 \mathrm{~cm}$ con sensibilidad y $100 \%$ de especificidad.

\section{DISCUSIÓN}

A pesar del gran número de pacientes obesos ingresados a la UCI, existen pocos trabajos o guías publicadas que especifiquen el correcto manejo del destete de VMI. Actualmente, no existe en la literatura internacional un predictor fidedigno de éxito para el retiro de la ventilación mecánica en este grupo de pacientes; por ello, el reto consiste en encontrar un predictor que nos dé sensibilidad y especificad de $100 \%$. Martino y colaboradores, ${ }^{19}$ en un estudio multicéntrico que incluyó pacientes con obesidad extrema, encontraron un mayor número de días de ventilación mecánica. Esto puede ser explicado por la capacidad vital, capacidad pulmonar total y volumen funcional residual que se encuentra reducida hasta en $30 \%$ en los pacientes con obesidad mórbida, lo cual lleva a un aumento de la presencia de hipoxemia, formación de atelectasias y, por lo tanto, in-

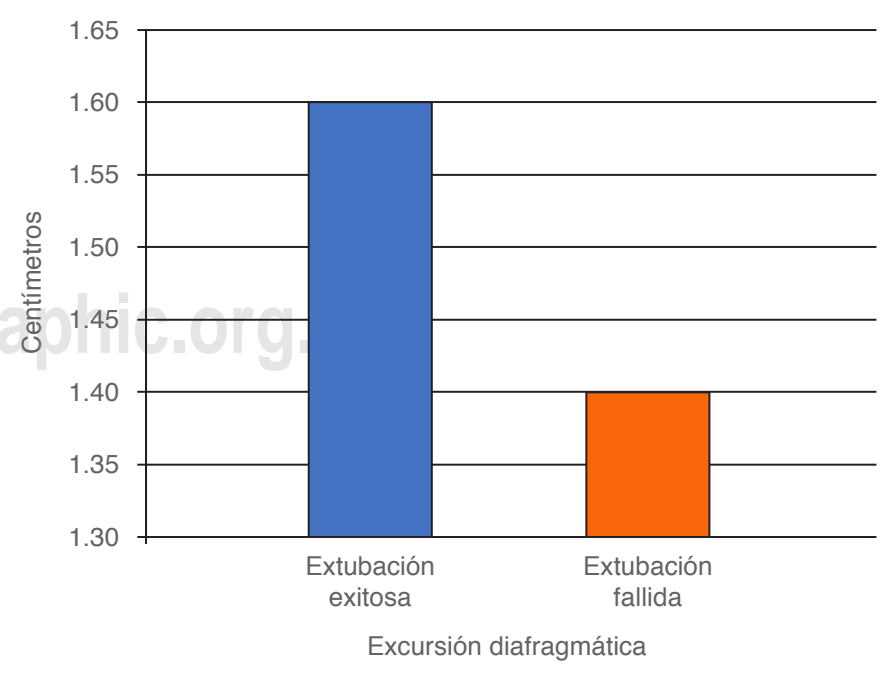

Figura 1: Excursión diafragmática de acuerdo con la extubación exitosa 0 fallida. 


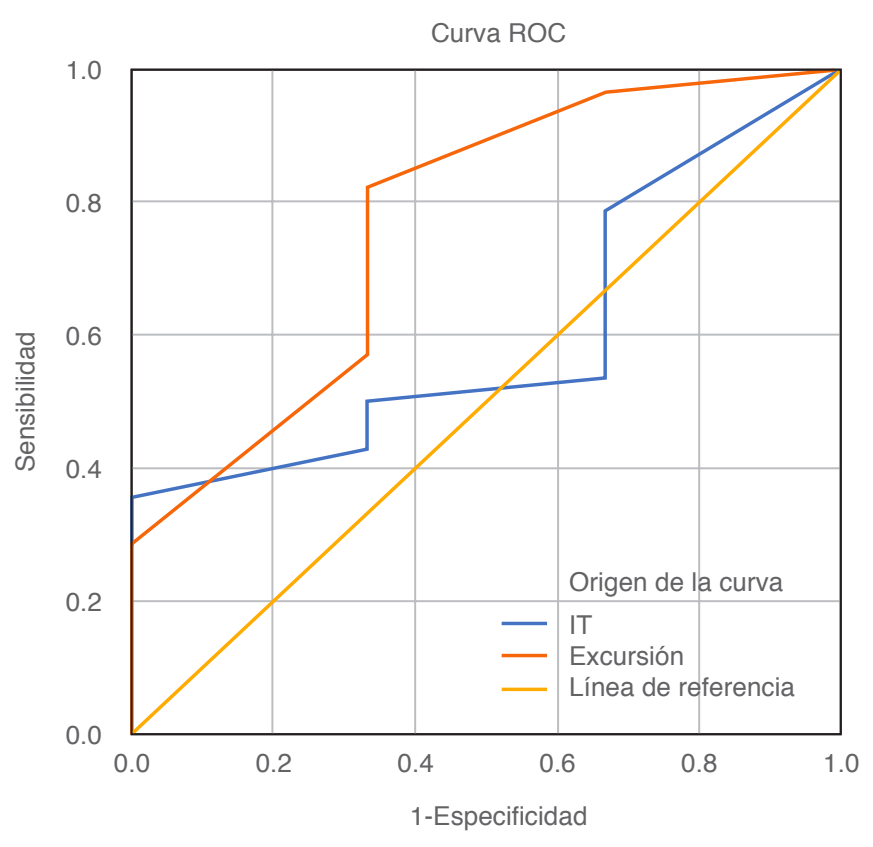

Los segmentos de diagonal se generan mediante empates.

Figura 2: Curvas ROC y AUC de la ED y el IVRS para el diagnóstico de extubación exitosa.

cremento del trabajo respiratorio y mayor dificultad en el retiro de la ventilación mecánica. ${ }^{20,21}$

En nuestra población, encontramos que son más los hombres que tienen obesidad, cuyo índice de masa corporal se reportó en promedio de $33.3 \pm 2.79 \mathrm{~kg} / \mathrm{m}^{2}$, y al hacer la comparación por las categorías de obesidad, se observó que en la clase 1 se encontró un $76.2 \%$ de los sujetos.

La principal causa de ingreso hospitalario fue por causas médicas. En la misma frecuencia, se encuentra dicha categoría como la principal en las mujeres; sin embargo, en los hombres, la causa quirúrgica fue la más presentada; en ambos sexos no se encontró un incremento en los días de ventilación.

La excursión diafragmática en la población total se encontró en $1.5 \mathrm{~cm}$ (0.3) (p50): en los hombres en 1.6 $\mathrm{cm}$, y en las mujeres en $1.4 \mathrm{~cm}$, y el punto de corte para la excursión diafragmática de $1.5 \mathrm{~cm}$, es decir, que se tiene una mayor probabilidad de presentar una extubación exitosa en comparación con aquéllos con cifras iguales o menores a $1.5 \mathrm{~cm}$ (RP 1.78), las cuales, de acuerdo con la literatura, se encuentran dentro de los parámetros; en individuos sanos, se han reportado de $1.8 \pm 0.3$ a $2.9 \pm 0.6 \mathrm{~cm}$ en hombres y de $1.6 \pm 0.3$ a 2.6 $\pm 0.5 \mathrm{~cm}$ en mujeres.

Para el retiro de la ventilación mecánica, Jiang realizó una evaluación de los movimientos diafragmáticos al medir el desplazamiento del hígado y bazo durante la prueba de ventilación espontánea, utilizando un punto de corte de $1.1 \mathrm{~cm}$ del desplazamiento hepático y esplénico. Éste demostró ser un buen predictor de desenlace en el retiro de la VMI, con una sensibilidad de $84.4 \%$ y una especificidad de $82.6 \%$, superando a los parámetros utilizados de manera rutinaria, como son el índice de respiración superficial y el Pimax. Los pacientes con un volumen tidal adecuado durante la PVE, que muestran una excursión diafragmática disminuida, tuvieron una mayor probabilidad de fracaso en el retiro que aquéllos con volumen tidal adecuado y una buena excursión diafragmática. Esto puede explicarse por el hecho de que el volumen tidal en la respiración espontánea está representado por una combinación de los músculos respiratorios sin que en ésta se reconozca la contribución del diafragma, mientras que la excursión diafragmática representa el resultado final de la fuerza del mismo músculo en combinación con la presión intratorácica e intraabdominal. ${ }^{22}$

Por su parte, $\mathrm{Kim}^{22}$ investigó la disfunción diafragmática por ultrasonido en modo $\mathrm{M}$ en 88 Unidades de Terapia Intensiva y encontró una prevalencia de $29 \%$ (por excursión diafragmática $<1 \mathrm{~cm}$ o movimientos paradójicos del diafragma). Los pacientes con disfunción diafragmática tuvieron un incremento en el número de intentos de retiro de la VMI y del mismo modo en los días de VMI. Estos resultados sugieren que la valoración ultrasonográfica del diafragma es útil para identificar a pacientes en riesgo de retiro difícil o prolongado, así como también es un predictor en el fracaso del retiro de la ventilación mecánica. ${ }^{19}$

Se pudo observar que la prevalencia de extubación exitosa en las mujeres fue mayor que en los hombres (69\%), por lo que las mujeres tienen mayor probabilidad de presentar una extubación exitosa en comparación con los hombres. No obstante, cuando nos enfrentamos a pacientes que conllevan un proceso de destete difícil, como es en esta población, ambos protocolos tienden al fracaso, principalmente por las características fisiopatológicas del paciente, las cuales contribuyen al fracaso en la PVE o durante el periodo próximo a la extubación.

El estudio mostró algunas limitaciones, como el reducido tamaño de la muestra que restringe la precisión del análisis estadístico, las limitaciones que implica la utilización del IMC para clasificar a estos pacientes y que puede ser alterado por la administración de líquidos en la fase de reanimación previa a su ingreso a la UCI.

\section{CONCLUSIONES}

La aplicación del IRS y de ED con los puntos de corte establecido en pacientes sin comorbilidades aplicados para esta población demostraron una mejor área bajo a curva para la ED comparada con el clásico IRS. Adicionalmente, se buscó un punto de corte para esta población, identificando el mejor punto de precisión, con mayor sensibilidad y especificidad $>1.9$ centímetros. 


\section{BIBLIOGRAFÍA}

1. De Jong A, Chanques $G$, Jaber S. Mechanical ventilation in obese ICU patients: from intubation to extubation. Crit Care. 2017;21(1):63.

2. Tafelski S, Yi H, Ismaeel F, Krannich A, Spies C, Nachtigall I. Obesity in critically ill patients is associated with increased need of mechanical ventilation but not with mortality. $J$ Infect Public Health. 2016;9(5):577-585.

3. Mogri M, Mador J. Mechanical ventilation of the obese patient. Critical Care Management of the Obese Patient. 2013.

4. Wadhwa A, Singh PM, Sinha AC. Airway management in patients with morbid obesity. Int Anesthesiol Clin. 2013;51(3):26-40.

5. Sengupta S, Chakravarty C, Rudra A. Evidence-based practice of weaning from ventilator: a review. ATOTW. 2018;(6):6.

6. Chen L, Gilstrap D, Cox CE. Mechanical ventilator discontinuation process. Clin Chest Med. 2016;37(4):693-699.

7. García-Arreola DAP, Alcántara-Morales MA. Obesidad: alteraciones fisiopatológicas y su repercusión anestésica. Rev Mex Anest. 2014;37(S1):198-206.

8. Hernández-López GD, Cerón-Juárez R, Escobar-Ortiz D, Graciano-Gaytán L, Gorordo-Delsol LA, Merinos-Sánchez G, et al. Retiro de la ventilación mecánica. Rev Asoc Mex Med Crit y Ter Int. 2017;31(4):238-245.

9. Imber DA, Pirrone M, Zhang C, Fisher DF, Kacmarek RM, Berra L. Respiratory management of perioperative obese patients. Respir Care. 2016;61(12):1681-1692.

10. Lu Z, Xu Q, Yuan Y, Zhang G, Guo F, Ge H. Diaphragmatic dysfunction is characterized by increased duration of mechanical ventilation in subjects with prolonged weaning. Respir Care. 2016;61(10):1316-1322.

11. Zambon M, Greco M, Bocchino S, Cabrini L, Beccaria PF, Zangrillo A. Assessment of diaphragmatic dysfunction in the critically ill patient with ultrasound: a systematic review. Intensive Care Med. 2017;43(1):29-38.

12. Qian Z, Yang M, Li L, Chen Y. Ultrasound assessment of diaphragmatic dysfunction as a predictor of weaning outcome from mechanical ventilation: a systematic review and metaanalysis. BMJ Open. 2018;8(9):e021189.

13. Ricoy J, Rodríguez-Núñez N, Álvarez-Dobaño JM, Toubes ME, Riveiro V, Valdés L. Diaphragmatic dysfunction. Pulmonology. 2019;25(4):223-235.
14. Carrillo G. Evaluación ultrasonográfica del diafragma en el enfermo grave. Rev Asoc Mex Med Crit y Ter Int. 2014;28(3):187194.

15. Schetz M, De Jong A, Deane AM, Druml W, Hemelaar P, Pelosi $\mathrm{P}$, et al. Obesity in the critically ill: a narrative review. Intensive Care Med. 2019;45(6):757-769.

16. Li C, Li X, Han H, Cui H, Wang G, Wang Z. Diaphragmatic ultrasonography for predicting ventilator weaning: A metaanalysis. Medicine (Baltimore). 2018;97(22):e10968.

17. Shashaty MG, Stapleton RD. Physiological and management implications of obesity in critical illness. Ann Am Thorac Soc. 2014;11(8):1286-1297.

18. Epstein SK. Destete de la ventilación mecánica: pruebas de preparación [monografía en Internet]. UpToDate. 2017.

19. Peñuelas O, Thille AW, Esteban A. Discontinuation of ventilatory support: new solutions to old dilemmas. Curr Opin Crit Care. 2015;21(1):74-81.

20. Umbrello M, Formenti P, Longhi D, Galimberti A, Piva I, Pezzi A, et al. Diaphragm ultrasound as indicator of respiratory effort in critically ill patients undergoing assisted mechanical ventilation: a pilot clinical study. Crit Care. 2015;19:161.

21. Montaño JA, Olvera GCI, Aguirre SJS, Camarena AG, Franco GJ. Pronóstico de la ventilación mecánica invasiva en el paciente obeso críticamente enfermo. Rev Asoc Mex Med Crit y Ter Int. 2018;32(5):277-284 .

22. Pérez-Calatayud AA, Carrillo-Esper R, Arch-Tirado E. Propuesta de evaluación cuantitativa en el protocolo ultrasonográfico para retiro de la ventilación mecánica invasiva (GMEMI score). Gac Med Mex. 2016;152(3):304-312.

Patrocinios y relación de conflicto de intereses: $\sin$ conflicto de intereses.

Correspondencia:

Beatriz Valerio Munguía

Calzada San Juan de Aragón Núm. 285,

Col. Granjas Modernas, 7460,

Alcaldía Gustavo A. Madero,

Ciudad de México.

E-mail: acua86_2007@hotmail.com 